Europhysics Letters

PREPRINT

\title{
An Information-Theoretic formulation of the Newton's Second Law
}

\author{
Mario J. Pinheiro \\ Department of Physics and Centro de Fisica dos Plasmas, \\ Instituto Superior Tecnico, Av. Rovisco Pais, \\ 1049-001 Lisboa, Portugal \\ PACS. 02.30.Xx - Calculus of variations. \\ PACS. 02.50. $\mathrm{CW}_{\mathrm{w}}$ - Probability theory. \\ PACS. 05.20.-y - Classical statistical mechanics.
}

\begin{abstract}
From the principle of maximum entropy for a closed system in thermal equilibrium, it is shown for the first instance, to exist a clear relation between total entropy $S$ (in terms of arrangements of particles) and the classical expression for the force acting on a particle in a rotating frame. We determine relationships between arrangement of particles and force in the case of the gravitational and elastic forces.
\end{abstract}

Introduction. - The notion of entropic force has been successfully applied to an increasing number of problems, e.g., to the calculation of forces acting at the ends of a single Gaussian macromolecule [1], the evocation of the geometric features of a surface as creating entropic force fields [2], and the attractive Coulomb force between defects of opposite type [3]. The purpose of the present paper is to present a new and unusual procedure, using a thermodynamical and mechanical framework, to obtain the (entropic) force acting over a particle in a rotating frame in terms of arrangement of particles.

Extremum Principle. - As is known, from Liouville theorem it is deduced the existence of seven independent additives integrals: the energy, 3 components of the linear momentum $\vec{p}$ and 3 components of the angular momentum $\vec{L}$. Let us consider an isolated macroscopic system $\mathcal{S}$ composed by $\mathrm{N}$ infinitesimal macroscopic subsystems $\mathcal{S}^{\prime}$ (with an internal structure possessing a great number of degrees of freedom, allowing the definition of an entropy) with $E_{i}, \overrightarrow{p_{i}}$ and $\overrightarrow{L_{i}}$, all constituted of particles of a single species of mass $m$. The internal energy $U_{i}$ of each subsystem moving with momentum $\vec{p}_{i}$ in a Galilean frame of reference is given by

$$
E_{i}=U_{i}+\frac{\vec{p}_{i}^{2}}{2 m}+\frac{\vec{L}_{i}^{2}}{2 I_{i}} .
$$

The entropy of the system is the sum of the entropy of each subsystems (and function of the their internal energy $U, S=S(U))$ :

$$
S=\sum_{i}^{N} S_{i}\left(E_{i}-\frac{p_{i}^{2}}{2 m}-\frac{L_{i}^{2}}{2 I_{i}}\right) .
$$

(c) EDP Sciences 
Energy, momentum and angular momentum conservation laws must be verified for a totally isolated system:

$$
\begin{aligned}
& E=\sum_{i=1}^{N} E_{i}, \\
& \vec{P}=\sum_{i=1}^{N} \overrightarrow{p_{i}},
\end{aligned}
$$

and

$$
\vec{L}=\sum_{i=1}^{N}\left(\overrightarrow{r_{i}} \times \overrightarrow{p_{i}}+\overrightarrow{L_{i}}\right)
$$

Here, $\overrightarrow{r_{i}}$ is the position vector of the ith part of the body (particle) relatively to a fixed frame of reference $\mathcal{R}$. It is necessary to find the conditional extremum; they are set up not for the function $S$ itself but rather for the changed function $\bar{S}$ :

$$
\bar{S}=\sum_{i=1}^{N}\left\{S_{i}+\vec{a} \cdot \overrightarrow{p_{i}}+\vec{b} \cdot\left(\overrightarrow{r_{i}} \times \overrightarrow{p_{i}}+\overrightarrow{L_{i}}\right)\right\},
$$

where $\vec{a}$ and $\vec{b}$ are Lagrange multipliers. The conditional extremum points are obtained for

$$
\frac{\partial \bar{S}}{\partial \vec{r}}=0 \frac{\partial \bar{S}}{\partial \vec{p}}=0
$$

At thermodynamic equilibrium the total entropy of the body has a maximum taking into consideration the supplementary eqs.( 3), (4) and ( 5).

The entropic force. - In the frame of the guiding principle briefly introduced [7], our main goal is to investigate if the information content of the entropy provides a knowledge of the motion of a system.

Usually it is used the relationship $d U=T d S-f_{r} d r-f_{\theta} d \theta-f_{z} d z$ (and interpreting here the $d^{\prime}$ as an exterior derivative 画) to obtain (along a given direction $v$ )

$$
\overrightarrow{\nabla U} v=T \nabla \vec{S}_{v}-\vec{f}
$$

On the above expression has its roots a kind of "entropic force" [1, ह]:

$$
\vec{f}_{\text {ent }}=T \vec{\nabla}_{r} S .
$$

We will write, instead of eq.( (7), the first and second principle combined in a differential form (in cylindrical coordinates):

$$
\vec{\nabla} U=\frac{T}{2} \frac{\partial \bar{S}}{\partial r} \overrightarrow{e_{r}}+\frac{T}{2 r} \frac{\partial \bar{S}}{\partial \theta} \overrightarrow{e_{\theta}}+\frac{T}{2} \frac{\partial \bar{S}}{\partial z} \overrightarrow{e_{\omega}}-\vec{f}
$$

It is noteworthy to introduce a factor of $\frac{T}{2}$, instead of $T$, to each degree of freedom. This procedure is necessary to eliminate a factor 2 which appear in front of the acceleration term. It means the scale of the "thermometer" is calibrated so as to give a number $\frac{T}{2}$, defined by

$$
\left(\frac{\partial U_{i}}{\partial S_{i}}\right)_{r_{i}}=\frac{T}{2} ; \quad\left(\frac{\partial U_{i}}{\partial S_{i}}\right)_{\theta_{i}}=\frac{T}{2} ; \quad\left(\frac{\partial U_{i}}{\partial S_{i}}\right)_{z_{i}}=\frac{T}{2} .
$$


This procedure is necessary to warrant the Equipartition Theorem [6] (see also Sect. 4.1).

Further on, for concreteness and simplicity, we consider a moving particle in a plane perpendicular to $\vec{e}_{\omega}$. In this case a simple calculation shows that the first term on the (right hand side) RHS of eq.(9) leads to

$$
\frac{T}{2}(\vec{\nabla} S)_{r}=\frac{\partial U}{\partial \vec{r}}+\frac{1}{2} \frac{\partial(\vec{u} \cdot \vec{p})}{\partial \vec{r}}+\frac{1}{2} \frac{\partial}{\partial r}[\vec{\omega} \cdot(\vec{r} \times \vec{p})]
$$

since $\overrightarrow{d r}=\vec{v} d t$ and $d \theta=\omega d t$. The second term of the RHS, gives

$$
\frac{T}{2}(\overrightarrow{\nabla \vec{S}})_{r}=\sum_{i}\left(\frac{\partial U_{i}}{\partial r_{i}}+m_{i} \frac{\partial v_{i}}{\partial t}\right)
$$

and, by the same token,

$$
\frac{T}{2}(\overrightarrow{\nabla S})_{\theta}=\sum_{i} \frac{1}{r_{i}} \frac{\partial U_{i}}{\partial \theta_{i}}
$$

Now, remark that using the transport equation

$$
\left(\frac{d \vec{v}_{i}}{d t}\right)_{\mathcal{R}}=\left(\frac{d \vec{v}_{i}}{d t}\right)_{\mathcal{R}^{\prime}}+\vec{\omega} \times \vec{v}_{i}
$$

we obtain

$$
\left(\frac{d \vec{v}_{i}}{d t}\right)_{\mathcal{R}}=\left(\frac{d \vec{v}_{r e l}}{d t}\right)_{\mathcal{R}^{\prime}}+\frac{d}{d t}\left(\vec{\omega} \times \vec{r}_{i}\right)_{\mathcal{R}}+\vec{\omega} \times \vec{v}_{\text {rel }} \text {. }
$$

Inserting into eq.(9) the previously deduced eqs.( 12 15), it follows that

$$
\vec{\nabla} U=\sum_{i}\left\{\vec{\nabla} U_{i}+m_{i} \dot{\vec{v}}_{r e l}+m_{i} \vec{\omega} \times\left(\vec{\omega} \times \vec{r}_{i}\right)+m_{i}\left(\dot{\vec{\omega}} \times \vec{r}_{i}\right)+m_{i}\left(\vec{\omega} \times \vec{v}_{r e l}\right)-\vec{f}_{i}\right\}
$$

Introducing

$$
\vec{\nabla}_{r_{i}} U_{i}=m_{i} \vec{a}_{\mathcal{R}, i}-m \vec{a}_{\mathcal{R}^{\prime}, i}
$$

which can be easily obtained by the outlined procedure and noting that $\sum_{i} \vec{\nabla} U_{i}=\vec{\nabla} U$, it is obtained the well known expression for the classical force in a rotating frame

$$
\vec{f}_{i}=2 m_{i}\left(\vec{\omega} \times \vec{v}_{r e l}\right)+m_{i}\left[\vec{\omega} \times\left(\vec{\omega} \times \vec{r}_{i}\right)\right]+m_{i} \dot{\vec{v}}_{r e l}+m_{i}\left(\dot{\vec{\omega}} \times \vec{r}_{i}\right)
$$

We conclude that the outlined information-theoretic formalism is able to deduce the classical force in a rotating frame (and hence Newton's second law); it makes the prediction that the gradient of entropy when multiplied by $\frac{T}{2}$ gives rise to a macroscopic force. We see that considering entropy as our primordial concept and with no arbitrary assumptions, it results conceptual and mathematical simplification.

Nevertheless, this classical force was obtained in a restrictive context that invokes a particle trajectory along an isentropic regime, obviously a limiting case of the behavior of matter. A new and very interesting physical situation could be explored considering a dynamical process in which a particle or system of particles evolves in nonequilibrium conditions.

The outlined argument could be reversed and allow the build up of a strategy to relate the force capturing the key properties of statistical ensembles.

For this purpose it is convenient to introduce the phase space $\Gamma$ of the system consisting in $N$ particles occupying a volume $V$ and energy between $E$ and $E+\Delta$ (in the microcanonical 
ensemble). Each point on $\Gamma$ represents a state of the system and the locus of all points in $\Gamma$ satisfying $H(p, q)=E$ defines the energy surface of energy $E$. The volume in $\Gamma$ space occupied by the microcanonical ensemble is

$$
\Sigma(E)=\int_{H(p, q)=E} d^{3} p d^{3} q .
$$

The entropy is defined by 8

$$
S(E, V) \equiv k \log \sum(E)
$$

Forces embed in microstates: an elementary approach. - If a macroscopic system is subject to an entropic force identified as the classical force under thermal equilibrium, it is legitimate to revert the argument and examine whether some microstates provides support for the existence of referenced kinds of forces. This question is illustrate subsequently.

Gravitational attraction. - Imagine a particle describing a circular motion at a distance $r$ away from the origin of a central force. The number of configurations in space associated with $r$ is $\Omega=4 \pi r^{2}$. Applying eq. ( 8 ) the entropic force results to be

$$
f_{r}=\frac{T}{2}(\overrightarrow{\nabla S})_{r}=\frac{k_{B} T}{r} .
$$

Taking into account the Equipartition Theorem, $\frac{1}{2} k_{B} T=\frac{1}{2} m v_{r}^{2}$, when thermal equilibrium prevails. Hence, the known expression for centrifugal force is found

$$
f_{r}=m \omega^{2} r
$$

Notice that the term of mass was introduced via Equipartition theorem. From this the expression for the force becomes (unfortunately, the symbol for period and absolute temperature are identical)

$$
f_{r}=m\left(\frac{4 \pi^{2}}{T^{2}}\right) r=m \frac{4 \pi^{2} r^{3}}{T^{2}} \frac{1}{r^{2}} .
$$

Recall that according to Kepler law, all the planets on the solar system have the same ratio $\frac{r^{3}}{T^{2}}=K$ :

$$
f_{r}=4 \pi^{2} K \frac{m}{r^{2}}
$$

Instead, Newton wrote the above equation on the form

$$
f_{r}=\left(\frac{4 \pi^{2} K}{M_{S}}\right) \frac{M_{S} m}{r^{2}}=G \frac{M_{S} m}{r^{2}}
$$

defining $G=\frac{4 \pi^{2} K}{M_{S}}$ as the gravitational constant. Therefore, the gravitational force is retrieved, but in the present model on the ground of a different viewpoint, giving a new evidence to an old law of physics.

Elastic spring. - The restoring force in a piece of stretched rubber can be interpreted in terms of a freely orientating Gaussian macromolecules containing N segments without volume, each with equal length $l$ and remaining at a vector distance $\vec{r}$. It can be shown that the number of different molecular configurations which are likely to occur is given by 
$\Omega(l) \propto b^{3} \pi^{-3 / 2} \exp \left(-b^{2} r^{2}\right)$ (here, $\left.b=\frac{3}{2} N l^{2}\right)$ [1, [5], from where it is obtained, following the same line of though as in the above example, the elastic force

$$
f_{r}=-k_{B} T b^{2} r
$$

This is the first approximation of a restoring force. Normally, as resulting from the law of elastic deformation (Hooke's law) $f=-k r$ and so the elastic constant should depend on the temperature as well as on structural parameter of the spring.

Conclusion. - The principle of maximum entropy and its mathematical model is a promising tool for treatment of new physical problems. Here, it was presented an unusual method to show that the classical force acting on a particle of mass $m$ in a rotating frame (and hence Newton's second law) can be obtained making use of the entropy concept. Particle's portrayal in space-time issues from the interrelationship of processes and information. This procedure could provide a direction toward an amazingly rich subject.

I wish to thank Prof. Paulo Sá for critical reading of the manuscript.

\section{REFERENCES}

[1] Richard M.Neumann, Am. J. Phys., 48(5) (1980) 354.

[2] A.D.Dinsmore, D.T.Wong, Philip Nelson and A.G. Yodh, Phys. Rev. Lett., 80(2) (1998) 409.

[3] Cristopher Moore, Mats G. Nordahl, Nelson Minar and Cosma Shalizi, arXiv:condmat, 9902200 v2 20 Feb (1999).

[4] Charles W. Misner, Kip S. Thorne and John Archibald Wheeler, Gravitation (W.H.Freeman \& Company, San Francisco) 1973.

[5] J.H. Weiner, Am. J. Phys., 55(8) (1987) 746

[6] Let us reduce our argument to one dimension. The force acting over a particle is $F=\frac{k X}{r}$, and so the work done along $r$ is $E=F r=k X$. In order the Equipartition Theorem be verified this must be equal to $\frac{k T}{2}$ and so $X=\frac{T}{2}$.

[7] Landau and Lifschitz, Physique Statistique (Mir) 1960

[8] K. Huang, Statistical Mechanics (John Wiley \& sons, New York) 1963 